\title{
Ratio-Recording Spectroradiometer
}

\author{
Harry K. Hammond III, Warren L. Holford, and Milton L. Kuder
}

(January 26, 1960)

\begin{abstract}
A ratio-recording spectroradiometer has been constructed primarily for determining the relative spectral irradiance from fluorescent lamps in the visible spectrum. Radiant flux from a test source and a comparison source is transmitted or reflected by separate diffusers. The irradiance from each diffuser is sampled alternately of the order of 100 times a second by a double prism monochromator with cam-linearized wavelength drive from 360 to $760 \mathrm{~m} \mu$. A 14-stage multiplier phototube with S-20 response is used with an electronic gate and integrator circuits to compare the spectral irradiances from the two sources. When the phototube outputs are unequal, a servo unit adjusts apertures in each beam to equalize them. The measured parameter is the amount of adjustment required for equalization at each wavelength. The apertures are adjusted by a cam coupled to a pen which records the ratio on a three-cycle logarithmic strip chart. The instrument requires about 8 minutes to record the spectrum at $10 \mathrm{~m} \mu$ per inch. An expanded scale of $1 \mathrm{~m} \mu$ per inch is used for evaluating the energy in the spectral lines. The speed of wavelength scan may be made inversely proportional to the unbalance signal, if desired, to provide ample time to record spectral lines accurately. Symmetry of beam treatment is demonstrated by interchanging test and comparison sources and recording the inverse ratio.
\end{abstract}

\section{Introduction}

In order to calibrate fluorescent lamp color standards on a sound physical basis, the relative spectral irradiance from the lamp should be determined. These data, when weighted by the response functions of the CIE standard observer, yield chromaticity coordinates directly. A spectroradiometer has been constructed at NBS for the measurement of relative spectral irradiance with particular regard to the problem of fluorescent-lamp measurement.

Spectrometers for dispersing light into its spectral components have been commercially available for a long time. However, a universal design has not been adopted for converting a spectrometer into a spectroradiometer because of the varied requirements of particular laboratories. A recording spectroradiometer was built by Zworykin in 1939 [1]. ${ }^{1}$ Ten years later, Studer and Jacobsen reported the design of a spectroradiometer which was available commercially for a time [2]. In 1957, however, no spectroradiometer was commercially available; so it became necessary to design one to meet our requirements.

\section{Requirements}

The general requirements of a spectroradiometer are simply stated. Radiant flux from a source must be dispersed into its spectral components and evaluated with a suitable detector and read-out device to provide relative spectral irradiance data. Accurate measurements require a high degree of spectral purity, but sufficient energy must be trans-

${ }^{1}$ Figures in brackets indicate the literature references at the end of this papr. mitted by the dispersing element for precise evaluation by the detector. A practical compromise between resolution and sensitivity is necessary, as very high resolution would permit only infinitesimal energy to be transmitted to the detector. The instrument response must be appropriate to record accurately the peaks of sharp spectral lines. It must be able also to average the light from a modulated source, such as a fluorescent lamp operated on alternating current.

For the purpose of evaluating fluorescent lamp chromaticity, a photometric accuracy of 1 percent is considered adequate. If the photometric errors are truly random, then the chromaticity coordinates computed from the spectral radiance data will be uncertain by much less than this amount.

\section{Basic Components}

Monochromator. Both prism and grating monochromators are available for dispersing light into a spectrum. Grating monochromators are attractive because the dispersion is almost constant over a practical range of wavelengths. This permits a direct connection to a linear wavelength scale and provides a pass band of constant spectral width for fixed mechanical slits. Gratings, however, have the disadvantages of overlapping orders, higher stray light, and possible ghosts. Stray radiant flux in any dispersion system is minimized by double dispersion. In a good double prism monochromator, the stray flux will be several orders of magnitude below the radiant flux in the pass band. After some investigation of monochromators available at a moderate price, a Carl Leiss double monochromator with dense flint glass prisms was selected. Dispersion data are 
shown in figure 1 . The three bilateral slits have straight sides $10-\mathrm{mm}$ long and are set independently. Data are taken with constant mechanical slits; hence the pass band is a function of wavelength. For measurement of fluorescent lamps, the entrance and exit slits are frequently set at $0.5 \mathrm{~mm}$ with the middle slit at $0.6 \mathrm{~mm}$. For these conditions, the pass band at $546 \mathrm{~m} \mu$ is just under $3 \mathrm{~m} \mu$ when evaluated as the width of the mercury line at half height.

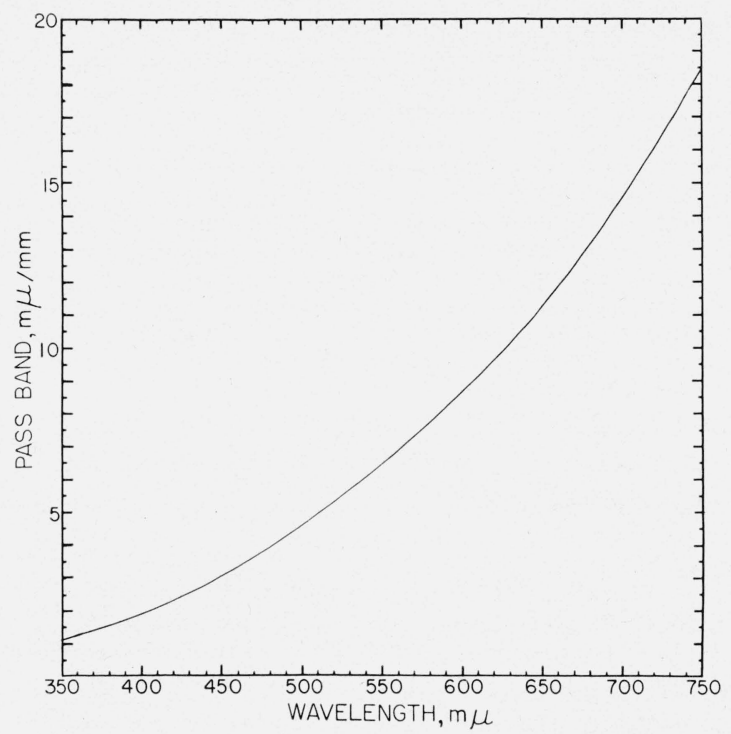

Figure 1. Dispersion data for spectroradiometer. Carl Leiss double monochromator with dense flint glass prisms.

The original nonlinear prism drive, consisting of sector and worm, has been disengaged. The new drive consists of a system of levers including an 18in. arm that follows a specially constructed cam with radii ranging from 3 to 7 in. The arm, cam, and associated gear train render the wavelength scale linear over the range 360 to $760 \mathrm{~m} \mu$.

Detector. The low level of radiant flux passing through the exit slit of the monochromator requires that a highly sensitive detector be employed. The detector should also provide good response over the entire range of spectrum to be measured. The equivalent noise input should be low to obtain a high signal-to-noise ratio for weak signals. The detector selected was a 14-stage multiplier phototube, RCA developmental type C7237, now designated as type 7265 . This tube is an end-on type with S-20 response, peaking in sensitivity at approximately $420 \mathrm{~m} \mu$ and maintaining 20 percent of the peak response at $700 \mathrm{~m} \mu$.

Recording System. Some spectroradiometers have been designed to use the output of the detector as the measured parameter. Instruments can be designed, however, that are essentially independent of the response characteristics of the phototube and the spectral transmittance of the monochromator. Considerations involved in this type of system have been published by Mitsuhashi in 1955 [3], and an instrument utilizing these principles was reported by MacAdam in 1953 [4] and described in detail in 1958 [5]. Our design was developed independently, but is similar in many respects to those cited above. Measurement is accomplished by rapidly comparing the flux from a standard source with that from a test source as the spectrum is scanned and optically attenuating the beams until the two have equal flux. For this system, the measured parameter is the setting of the beam attenuator. Attenuator settings are recorded on a strip chart, giving a plot of ratios of relative spectral irradiance from the two sources as a function of wavelength.

Attenuator System. There are two requirements that should be considered in the design of an attenuator system. The geometric distribution of flux incident on the entrance slit of the monochromator should not be appreciably affected by the attenuator, and the calibration of the system should not be a function of wavelength. The latter requirement cannot be met by optical wedges or polarizing filters. The former requirement cannot be met by a single adjustable aperture but for practical purposes is satisfied by an array of apertures. To compare precisely sources whose spectral irradiance ratios vary over a wide range, the aperture arrangement should be chosen so that the transmittance will be a rapidly changing function of displacement. Our basic attenuator design consists of an array of 25 square openings, $1 / 10 \mathrm{in}$. on a side, etched in metal foil, and arranged as shown in figure 2. Each attenuator consists of a pair of arrays, one fixed and one movable on an arc whose radius is about $4 \mathrm{in}$. The change in area of the openings, and therefore the transmittance, is proportional to the second power of the displacement of the movable array. When arranged so that the attenuator in one beam opens as the other closes, the transmittance ratio is a fourth-power function of the displacement drive, provided the flux through the attenuators is relatively uniform.

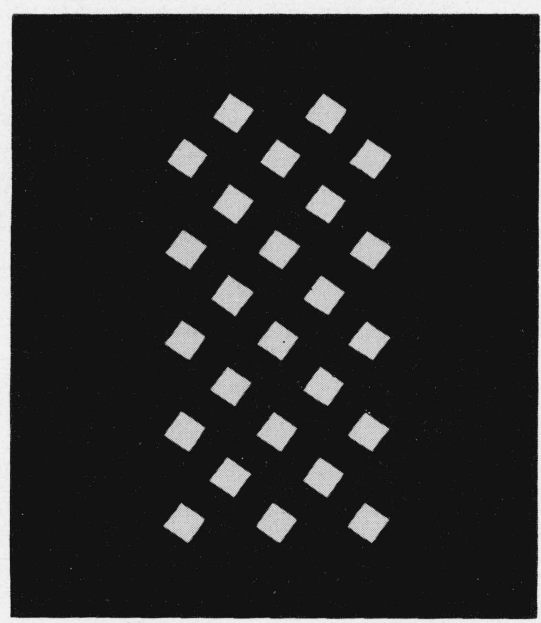

Figure 2. Attenuator array. 


\section{Entrant Beam Geometry}

The entrant beam geometry is shown in figure 3 . The rotating sector beam switch, consisting of a wheel with four sectors of alternate apertures and mirrors, alternately passes light from each source into the monochromator. The irradiance from each lamp may be sampled at a rate which is adjustable from 20 to 120 times a second. Compartments for filters are located in each beam for use when desired. The optical attenuators are located in each beam and operate in opposition, one opening while the other is closing. The two light sources are external to the instrument case and, therefore, can be easily changed.
The cone of rays at the entrance slit of the monochromator required to fill the collimating mirror was determined by rear projection of a source placed at the exit slit. To prevent the monochromator from giving a different weighting to rays from different sources, it is necessary that they both subtend the same solid angle at the entrance slit, or better, that they fill the maximum solid angle of the monochromator optics uniformly. To achieve this goal at some distance from the slit requires that the sources have either relatively large areas of uniform radiance or that they uniformly irradiate reflecting or transmitting diffusers of appropriate size.

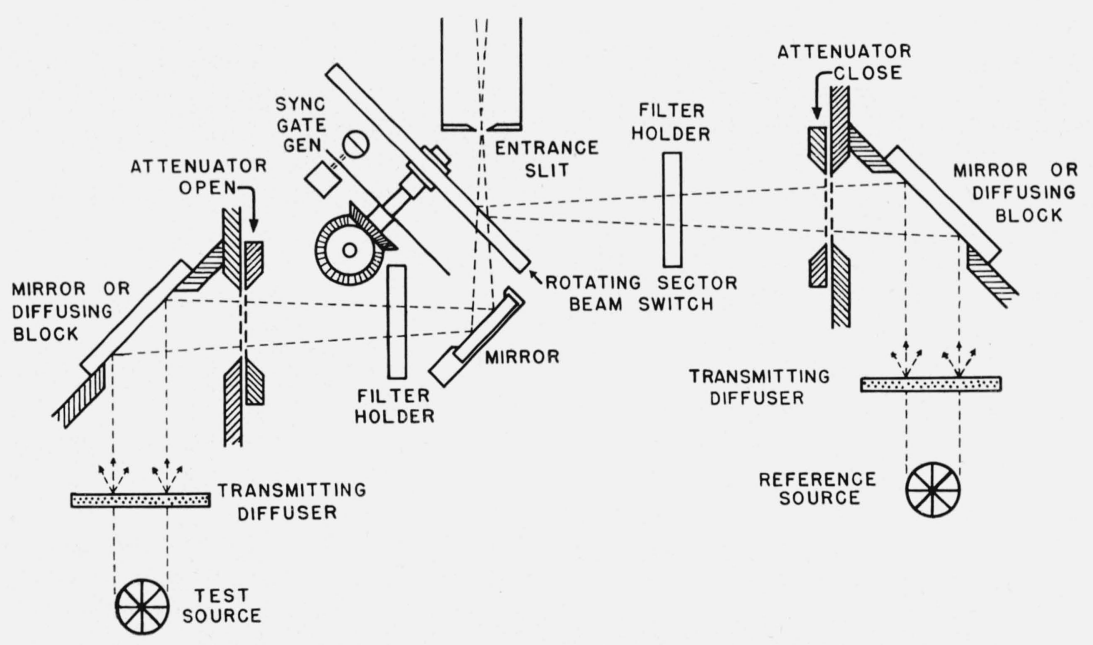

Figure 3. Diagram of entrant beam geometry.

\section{Photometric Scale}

In order to achieve the desired photometric scale on the strip chart, a precision linkage has been installed between the attenuators and the servo drive mechanism. The two movable elements of the attenuators are displaced simultaneously by two identical eccentric disks on a common shaft. The shaft extends to the strip chart recorder where it terminates in a cam-following lever. A relatively simple cam, driven by the recorder servomotor, converts the fourth power function to the desired logarithmic function. The location of cam and follower are shown in figure 4 . The recorder plots the area ratio of the attenuators on a logarithmic scale. Cams may be interchanged by the removal of one thumb nut to provide different photometric scales. For fluorescent-lamp measurements, however, the cam consistently used provides a three-cycle log scale on the 10-in. width of the strip chart. The intense lines of the mercury spectrum are easily accommodated with this scale.

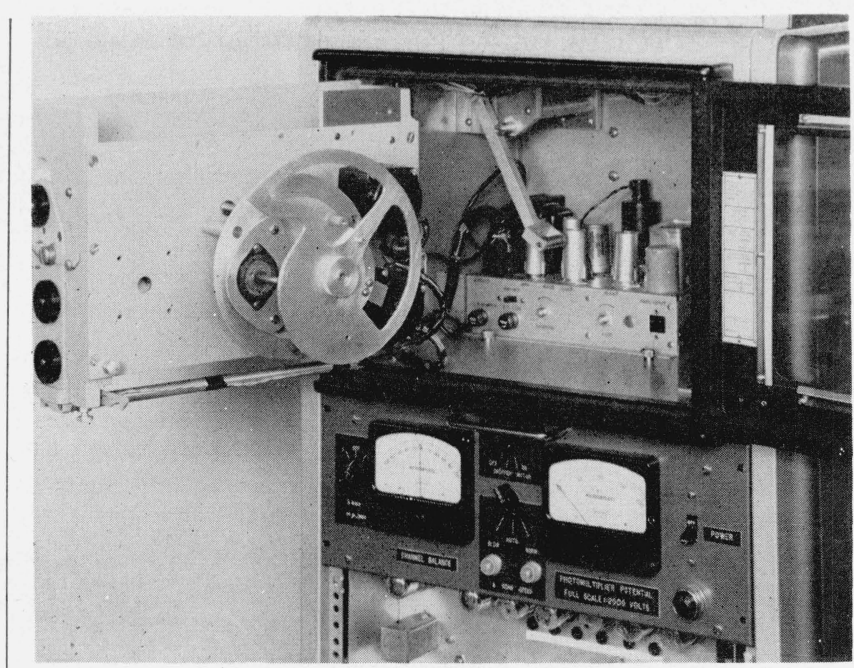

Figure 4. Photometric cam provides 3-cycle log scale. 


\section{Electronics}

The dynode voltage supplied to the phototube is continuously adjusted automatically to maintain constant average anode current. This feature provides constant detector sensitivity as spectral irradiance from a source decreases, until a minimum photon level is reached and maximum gain is applied. This feature also prevents the phototube from giving erroneous balance indications due to anode current saturation caused by high gain at high flux levels. Phototube damage due to excessive gain at such levels is also prevented by this automatic voltage adjustment.

Signals from two sources are separately supplied to two integrating networks by electronic gates as shown in the block diagram of figure 5. The gating signals are generated by sets of auxiliary light sources and phototubes. One set is used for gating the test source signals, another for the signals from the reference source. An opaque disk on the beam switch motor shaft has precisely positioned apertures which produce the gating signals. The gate is opened after the test or reference flux falls on the detector and is closed before the flux is switched off. The gated signals are essentially square waves. The integrator circuit and subsequent filter are designed to provide a smooth direct-current signal to the conventional servoamplifier of the recorder. The integrating network has a time constant of about $1 / 2$ sec. The integrator also serves to average signal variations due to modulated radiant flux from an a-c operated fluorescent lamp as well as noise pulses from the phototube. When the integrated signals from the two sources are unequal, the servo drives the attenuators until a balance is achieved.

The magnitude of the unbalance signal is also used to control the rate of wavelength scan. One motor drives the prisms and the recorder chart and thus wavelength correlation is maintained between the spectrum and the chart regardless of the scanning rate. The speed of the drive motor is modulated according to the magnitude of the unbalance signal. This modulation is roughly proportional to the

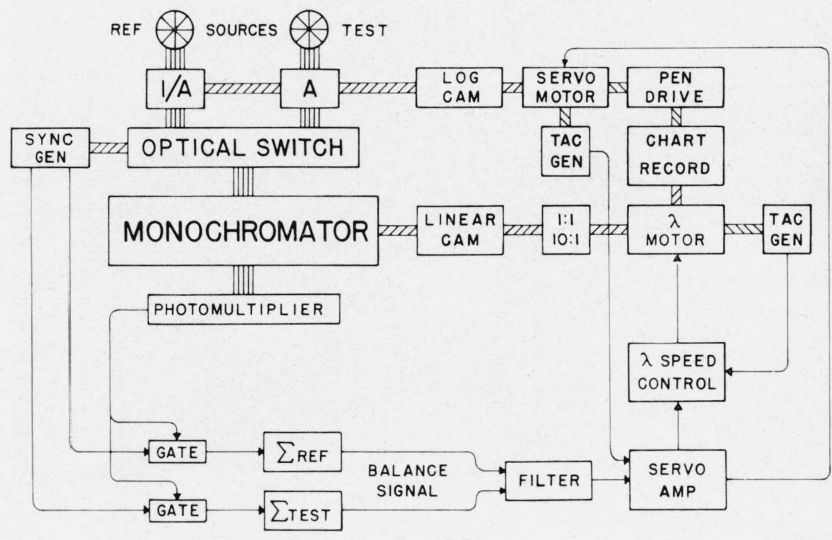

Figure 5. Block diagram of instrumeni components. reciprocal of the first derivative of the curve on the chart. When the curve is flat the rate of scan is maximum. As the slope of the curve begins to change the rate of scan is automatically reduced to prevent inaccurate recording imposed by phase lag in the servo response. This feature insures the faithful recording of the peaks of sharp spectral lines.

Tachometer generators are used in conjunction with both the wavelength drive and photometric balance motors. One generator provides motor speed information to the wavelength-scan speedcontrol unit. The other generator provides velocity damping for the balance motor.

The wavelength drive has been equipped with auxiliary gears and electric clutches to provide two chart scales, $1 \mathrm{~m} \mu /$ in. or $10 \mathrm{~m} \mu /$ in., merely by energizing the proper clutch. The expanded scale of $1 \mathrm{~m} \mu /$ in. is most useful for evaluating the flux in spectral lines. When a modulated scanning rate is not desired, a manual switch provides for selection of one of two uniform scanning rates. With a chart scale of $10 \mathrm{~m} \mu /$ in. the approximate times required to scan the visible spectrum at each speed are $8 \mathrm{~min}$ and $30 \mathrm{~min}$.

\section{Meters and Controls}

The front panel of the console is shown in figure 6 . The wavelength motor shaft has been extended to the front of the instrument to permit convenient manual setting to any desired wavelength. Two dials are used, one reading hundreds and tens of millimicrons, the other reading units and tenths, so that wavelengths can be read easily to better than $0.1 \mathrm{~m} \mu$. Calibrations with spectral-line sources, however, show that the original cam has wavelength-scale errors of as much as $0.2 \mathrm{~m} \mu$ below $580 \mathrm{~m} \mu$ and somewhat larger errors at longer wavelengths.

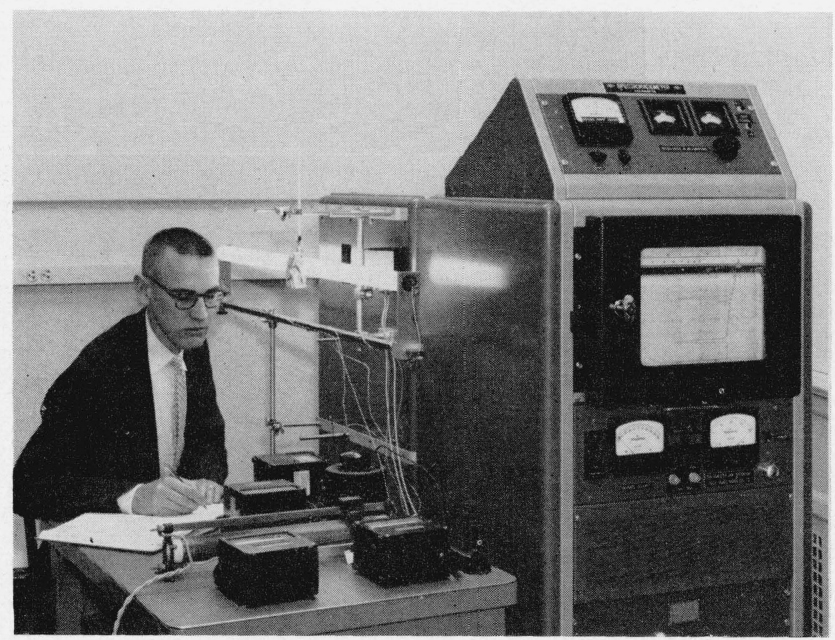

Figure 6. Mr. Holford records elecirical parameters while instrument records spectral irradiance of fuorescent lamp relative to an incandescent lamp.

Photograph was made prior to use of external reflecting diffuser. 
The channel balance meter in the lower left indicates the difference voltage between the test and reference signals. During operation, the difference voltage indications will be very small if the servo balance unit is operating properly.

The meter on the right indicates the dynode supply voltage to the phototube. Since the average anode current is held constant automatically, a lower flux level requires a higher dynode voltage. As the dynode voltage is raised to the maximum permissible value, the signal-to-noise ratio is lowered slightly. Further reduction in flux level results in a noisy signal and consequently produces an erratic recorder trace. Thus the dynode-voltage meter provides an indication of whether there is enough flux to permit a reliable ratio determination.

The meter at the upper left also provides indications proportional to the dynode voltage. This meter, however, is provided with zero suppression and sensitivity controls so as to provide an indication of the location of a spectral line when manually manipulating the wavelength knob during wavelength calibration.

Switches for controlling power, speed of scan, and wavelength scale are conveniently located on the front panel. Pushbuttons to provide fast travel of the wavelength drive in either the forward or backward direction are located near the wavelength dials. Potentiometers for setting electrical characteristics in the balance circuit and in the speed control unit to provide optimum instrument performance are located behind the panel below the two meters.

\section{Calibration}

During construction of the instrument, particular care was given to the design of the components whose calibration directly affected the instrumental accuracy. Mechanical linkages in the wavelength drive and photometric system were held to close tolerances and materials chosen were those least likely to wear or change with time. Several hundred hours of instrument operation have revealed no changes due to wear. Frequent wavelength calibrations using narrow slits of $0.15 \mathrm{~mm}$ reveal that wavelength scale calibration is reproducible to better than $0.1 \mathrm{~m} \mu$.

The photometric cam is calibrated by utilizing ratios of 2 to 1 . Two lamps of equal intensity, as measured by the spectroradiometer, irradiate the diffuser at one entrance port. A reference lamp illuminating a diffuser at the second port is compared with each of these lamps in turn to assure their equality, and then compared with the sum of the two lamps. The resulting irradiance ratio is precisely 2. The wavelength drive is not operated during the photometric calibration. The three-cycle $\log$ chart is adequately covered by nine steps of ratios of 2 to 1 . The intermediate calibration points are interpolated with data based on micrometric measurements of the cam radius. The reproducibility of photometric calibrations over the usable portion of the cam is about 0.3 percent.

\section{Performance Tests}

Tests have been made to evaluate the performance of the spectroradiometer. The investigation of possible systematic errors has been aided by the fact that the test lamp may be operated at either of the two entrance ports with the reference lamp at the other. Because photometric accuracy is determined by the geometric distribution of the radiant flux at the attenuators and the calibration of the photometric cam, the exchange of entrance ports produces reciprocal conditions which provide internal checks on instrument performance. A small discrepancy in fluorescent-lamp measurements was revealed when the reference and test sources were interchanged. This caused an investigation to be made of possible systematic errors or bias in the instrument. The electronic circuits for each channel were interchanged, but these circuits were not the cause of the biased results. The electronic system was further vindicated when practically identical spectral-irradiance data were recorded from a fluorescent lamp operating first on alternating current and then on direct current. A part of the small discrepancy was attributed to a bias in the servo unit. Equal, but opposite voltages to the servoamplifier did not produce equal torques at the servomotor because the voltages on the motor windings were not exactly $90^{\circ}$ out of phase. The effect was noticeable only when the signals were relatively weak. A trimmer capacitor placed across one of the motor windings produced the required phase relationship for balanced operation of the servo motor.

Further investigation revealed that interchange of reference and test sources introduced no discrepancy when the test source geometry is similar to that of the calibration source. A fluorescent lamp, however, has a geometry quite different from that of the incandescent lamp standard. The discrepancy between results obtained by interchanging test and reference sources was traced to the nonuniform irradiation of the diffusing block placed at an angle of $45^{\circ}$ to the axis of the fluorescent lamp. The nonuniform irradiance distribution from the incandescent standard was included in the calibration, but the entrance port acted as a field stop for the fluorescent lamp, thereby producing a different irradiance distribution.

In an attempt to eliminate this difficulty, the reflecting diffusers were replaced with mirrors, and ground-glass transmitting diffusers were mounted parallel to the axis of the fluorescent lamp. Ground glass, however, acts like a large number of tiny prisms and thus deviates shortwave energy more than longwave energy. This differential diffusion can be tolerated when comparing sources of the same size. When test and standard sources are different in size, as is the case with fluorescent and incandescent lamps, then different amounts of radiant flux are scattered out of the beam at each wavelength and erroneous values of relative spectral irradiance are obtained. Further consideration of the use of reflecting diffusers indicated that the problem of non- 
uniform irradiance of the test diffuser could be solved by relocating it external to the instrument in such a position as to obtain uniform irradiance from the fluorescent lamp.

\section{Modes of Operation and Applications}

Accurate determination of the ratios of relative spectral irradiance from a test and a standard source by direct comparison requires similar treatment of each beam. Light from each source must traverse equivalent paths to prevent erroneous results caused by spectral selectivity of the system. No evidence of asymmetry has been discovered, but the majority of measurements have been made by using a substitution technique. In this technique a reference source is calibrated by operating the standard source on the test side. This procedure has the disadvantage of requiring periodic calibration of the reference source, but it has four advantages: (1) The standard and test beams travel the same paths; (2) the life of the standard lamp is conserved by operating it only to calibrate the reference source; (3) the reference source may be a low-wattage, short-life lamp which produces less heating of the air in the vicinity of the fluorescent lamp than does the usual 500-w projection lamp regularly supplied as a color-temperature standard; and (4) the spectral irradiance from the reference source can be modified by an uncalibrated filter. The use of a blue filter reduces the flux from the reference source at the red end of the spectrum, thereby keeping the flux ratio of fluorescent to reference lamp on scale. If a filter were not used, the ratio of flux of incandescent standard at $2854^{\circ} \mathrm{K}$ to reference source would plot as a horizontal line throughout the spectrum and the curve for fluorescent lamp "B" would be off the bottom of the scale beyond $700 \mathrm{~m} \mu$. See figure 7 .

The spectroradiometer was built primarily for the measurement of fluorescent lamps. However, the instrument can measure the relative spectral irradiance from any source or source-filter combination that will provide a minimum illumination of about $1 \mathrm{fc}$ on the diffuser. The transmittance of filters can be obtained by computing the ratio of relative spectral irradiance from the source-filter combination to that from the source alone. A "100 percent" curve is recorded as the irradiance ratio of two sources. If the sources are identical, the irradiance ratio will be constant throughout the spectrum, and the plot will be a line parallel to the wavelength axis. Transmittance data may be recorded by inserting the filter in either of the two beams. By measuring the filter in each beam and averaging the result, any inaccuracies in the photometric-cam calibration above and below the 100 percent curve will be averaged. Comparison of two filters may be made directly by inserting one in each beam.

Reflectance data for a specimen illuminated with any desired flux geometry may be obtained by computing the ratio of spectral radiance of the specimen

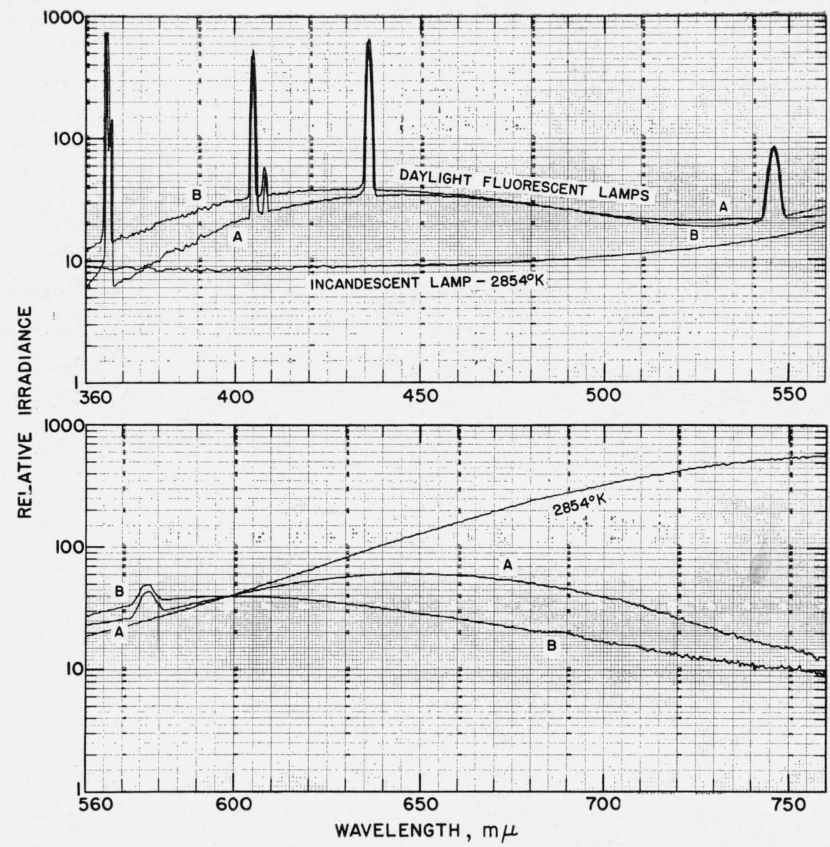

Figure 7. Curves obtained for two daylight fluorescent lamps and an incandescent lamp at $2854^{\circ} \mathrm{K}$.

These curves are ratios of the irradiance from the test lamp to that from an incandescent reference lamp and Corning blue-green filter type 4308 . Filter is used to limit irradiance-ratio range of lamps to two log-cycles. Daylight fluoresused to limit irradiance-ratio range of lamps to two log-cycles. Daylight fluores-
cent lamps usually do not differ by as much as shown here. Curve A was obcent lamps usually do not differ by as much as shown here. Curve A was ob-
tained with an old lamp utilizing a discontinued bery!lium phosphor. Curve B tained with an old lamp utilizing a discontinued bery!lium phosphor. Curve B
was obtained with a lamp from current production utilizing a phosphate phosphor. Low values of irradiance from both test and reference sources below $400 \mathrm{~m} \mu$ and above $700 \mathrm{~m} \mu$ cause the trace to be noisy.

to that of a standard diffuser. Luminescent or fluorescent materials can also be measured if they have several inches of uniform luminance and if the luminance is of the order of $1 \mathrm{fL}$.

The chart drive incorporates several flexible features. As previously mentioned, data may be recorded with wavelength scales of $10 \mathrm{~m} \mu /$ in. or $1 \mathrm{~m} \mu /$ in. The recorder chart can also be driven without advancing the wavelength so as to produce a record of the radiant flux of a spectrally homogeneous signal as a function of time.

Cams have been designed to provide 1 or $3 \mathrm{log}$ cycles on the 10-in. strip chart. More precise data, however, may be obtained by reading directly two dial scales; one, a coarse scale, is on the pen-cable drum and the other, a fine scale, is on the servo motor shaft. The precision of photometric readings is approximately 0.1 percent on the dial scales.

\section{Discussion}

Spectral-irradiance data for a light source can be obtained relative to an incandescent lamp of known color temperature in relatively short time with good precision. Reduction of data from the chart record, however, is time consuming and in addition appears to be the weakest link in the chain of data precision. As indicated above, dial readings of servo motor balance positions have been used for calibration and on other occasions to provide readings of highest preci- 
sion. Photometric calibrations can be made with a reproducibility of a few tenths of a percent, whereas chart readings are uncertain by about half a percent. Greater precision and increased speed of data reduction could be obtained by utilizing an electronic encoder to obtain decimal or binary digital readout on punched paper tape or other high precision recording means. Data in this form could then be supplied without transcription to a high-speed electronic computer to obtain chromaticity coordinates.

The authors express their thanks to Paul E. Allison for his contribution of skilled craftsmanship in the fabrication of mechanical parts for the instrument and to Jerome S. Laufer for his assistance in the selection of some of the components for the instrument.

\section{References}

[1] V. K. Zworykin, An automatic recording spectroradiometer for cathodoluminescent materials, J. Opt. Soc. Am. 29, 84-91 (1939).

[2] F. J. Studer and W. R. Jacobsen, Spectroradiometry, Gen. Elec. Rev. 52, No. 10, 34-9 (1949).

[3] H. Mitsuhashi, Some considerations of the balanced spectroradiometry, Sci. of Light 4, 61-71 (1955).

[4] D. L. MacAdam, Automatic recording spectroradiometer, J. Opt. Soc. Am. 43, 819A (1953).

[5] D. L. MacAdam, Continuously recording spectroradiometer, J. Opt. Soc. Am. 48, 832-40 (1958).

Washington, D.C.

(Paper 64C2-35) 